\title{
Scaling the Performance and Cost for Elastic Cloud Web Services
}

\author{
Monika Simjanoska, Marjan Gusev, Sasko Ristov and Goran Velkoski \\ Faculty of Computer Science and Engineering, Ss. Cyril and Methodius University, Skopje, Macedonia
}

\begin{abstract}
Cloud computing is the latest evolution of computing where the IT resources are offered as services following the "pay-per-usage" pricing model. Cloud's scalability feature causes variable price for resources governed by the cloud service providers. Therefore, the cloud customers' main interest is whether the performance scales to the price for the leased resources in the cloud. In this paper we analyze the variable server load impact on the performance and the cost of two web services that utilize memory and CPU resources. In order to determine the real cost of the rented CPU resources, we experimented with different number of concurrent messages with different sizes. The results concerning the memory demanding web service show that the lowest cost is obtained when the web service is hosted on two CPUs, whereas the results concerning the web service which additionally utilizes CPU show that the lowest cost is achieved when it is hosted on one CPU and linearly rises with the resources.
\end{abstract}

Keywords: cloud computing, web services, performance, resources, cost

\section{Introduction}

The cloud is a parallel and distributed computing system, which consists of a collection of interconnected and virtualized computers that are dynamically provisioned and presented as one or more unified computing resources. The resource provisioning is negotiated via service level agreements (SLAs) between the service providers and the consumers [1]. Cloud computing refers to the applications and services that run on a distributed network using virtualized resources and are accessed by common Internet protocols. It makes the long-held dream of utility computing possible with a pay-as-yougo, infinitely scalable, universally available system [2]. This usage-based pricing model offers several advantages, including reduced capital expense, a low barrier to entry, and the ability to scale up as demand requires, as well as to support brief surges in capacity [3]. A natural expectation from the pay-per-usage pricing model is that the performance gain scales to the monetary costs, i.e., the more resources are rented, the more performance is achieved. Cloud service providers (CSPs) guarantee the availability of the rented resources to the customers; however, a guarantee of scalable and sustainable performance is missing in the SLAs [4]. Hence, the problem of scalable performance is a very challenging field of study and is also beneficial for both the consumers and the CSPs.

In this paper, we conduct research to inspect the trade-off between the consumer's monetary costs for resources and the performance gain. Most of the related studies consider the CSPs' benefits, whereas the customers' expenses and privileges are not fully covered in the literature. In our research, we consider a single user case whose monetary costs for resources are proportional to the amount of rented resources. In order to simulate realistic occasions of renting different amount of resources, we prepared the cloud testing environments following the CSPs' pricing models presented in Tables 1 and 2, for Windows and Linux platform, respectively. These tables present the current offers for renting virtual machine (VM) instances, indicated in USD per rented hour. We present these offers as a proof that the linear pricing model is the current business model of the most common CSPs. The following VMs are analyzed: Windows Azure [5], Google Compute [6] and Amazon EC2 [7], as the most common and present on the market. 


\begin{tabular}{|c||c|c|c|c|}
\hline Type & 1 VM & 2 VMs & 4 VMs & 8 VMs \\
\hline \hline Windows Azure & 0,09 & 0,18 & 0,36 & 0,72 \\
\hline Google Compute & 0,145 & 0,290 & 0,580 & 1,160 \\
\hline Amazon EC2 & 0,091 & 0,182 & 0,364 & 0,728 \\
\hline \hline Scaling factor & 1 & 2 & 4 & 8 \\
\hline
\end{tabular}

Table 1. VM instance types and price simulation for Windows platform.

\begin{tabular}{|c||c|c|c|c|}
\hline Type & 1 VM & 2 VMs & 4 VMs & 8 VMs \\
\hline \hline Windows Azure & 0,06 & 0,12 & 0,24 & 0,48 \\
\hline Google Compute & 0,145 & 0,290 & 0,580 & 1,160 \\
\hline Amazon EC2 & 0,06 & 0,12 & 0,24 & 0,48 \\
\hline \hline Scaling factor & 1 & 2 & 4 & 8 \\
\hline
\end{tabular}

Table 2. VM instance types and price simulation for Linux platform.

To compare how the performance and cost scale, and to obtain sophisticated results, two web services are hosted in cloud environment with VM instances with the same platform, but different amount of hardware resources. The first web service is a memory demanding (Concat WS), which returns the concatenation of two strings, while the second (Sort WS) web service utilizes both the memory and CPU, returning the sorted concatenation of the two input strings. The Sort web service was discussed in our previous research [8], where we analyzed the web services' characteristics impact on the performance benefits. In this research we extend our analyses in more detail.

We focus our research in two directions:

1. How does the performance scale if the resources are scaled for the same server load;

2. Is there a region of server load where maximum performance is achieved by paying the same price.

The rest of the paper is organized as follows. In Section 2 we present a brief review of the related work. The testing methodology is defined in Section 3 and the results of the experiments are presented in Section 4. We evaluate the cost performance trade-off in Section 5 and discuss the obtained results in Section 6. Finally, we derive conclusions over the results and we present our ideas for a future research extension in the final Section 7.

\section{Related Work}

In this section we review recent research closely related to our field of interest.

The recent research of a "cost-efficient" cloud computing mostly examines the CSPs' costs for offering cloud computing solution. Either the authors investigate the cloud's energy and cost efficiency $[9,10,11,12]$, or they discuss other issues and challenges stating that with on-demand resource provisioning and utility based pricing, CSPs can truly maximize resource utilization and minimize their operating costs [13].

In this paper, we are interested in the customers' benefits of the on-demand resource provisioning and the pay-per-usage pricing model. Thereto, we aim to investigate if there is a case when the customer can achieve maximum performance with minimal costs. Similar research of this kind is presented by De Assuncao et al. [14], where the authors present several scheduling strategies for balancing between performance and usage cost. Their results show that some of the strategies result in a higher cost under heavy load conditions, whereas some showed a good ratio of slowdown improvement to the money spent for using cloud resources. Andrzejak et al. formulated a probabilistic model that enables a user to optimize monetary costs, performance and reliability, given the user's SLA constraints as resource availability and deadline for job completion [15]. Using their model, the users can achieve largest cost savings (for considered workload types) by using the high-CPU instance types instead of standard or high-memory instance types. The authors' contribution in [16] is developing a service that is able to perform the cost determination for scientific applications in cloud computing environments. Kondo et al. compare the performance and monetary costbenefits of clouds for computing applications, ranging in size and storage [17].

Considering the performance, using cache intensive algorithms in both single-tenant and multi-tenant cloud environments, Gusev and Ristov show how and when cloud computing can achieve even better performance than traditional environment for certain workload [18]. Another research for cloud's performance states 
that the cloud achieves smaller performance degradation for greater message sizes using a memory demanding web service, and also for greater message sizes and smaller number of concurrent messages for memory demanding and computation intensive web services [19]. In our previous research [20] we proved that the same web services achieved almost 10 times better performance when they were hosted in several VM instances with one CPU core, rather than in one huge VM instance with all CPU cores allocated.

As we presented the latest research related to the problem of scaling performance and cost in the cloud, we proceed with research methodology to find out if the performance rises linearly to the cost.

\section{The Methodology}

In this section, we developed an original methodology that includes technical details, appropriate cloud environment configuration, to include all three test cases with different number of resources, and the testing procedure itself. Considering the outcomes from the experiments we present relevant mathematical relations to obtain reliable results.

\subsection{Hardware Details}

As a testing environment we used client-server architecture deployed in the open source cloud platform OpenStack [21] using Kernel-based Virtual Machine (KVM) hypervisor to instantiate VM instances. The client and server node are installed with Linux Ubuntu Server 12.04 operating system. Hardware computing resources consist of Intel(R) Xeon(R) CPU X5647 @ $2.93 \mathrm{GHz}$ with 4 cores and 8GB RAM. The VM instances consist of Linux Ubuntu Server 12.04 operating system and Apache Tomcat 6 as the application server. To minimize the network latency (less than $1 \mathrm{~ms}$ ) we placed the client and the VMs in the same LAN segment [22].

\subsection{Web Services Description}

Both Concat and Sort web services are document style Java web services:
- Concat is a memory demanding web service that accepts two strings and returns their concatenation. It depends only on the input parameter size $M$ with memory complexity $O(M)$.

- Sort is a web service that accepts two strings and returns their alphabetically sorted concatenation. It is both CPU intensive and memory demanding. The memory complexity is $O(M)$ and time complexity is $O(M$. $\left.\log _{2} M\right)$. Sorting is realized with the merge sort algorithm.

\subsection{Environment Configuration}

In order to simulate various number of provided resources (CPU cores), we defined three different cloud environments presented in Figure 1:

- Test Case 1: VM instance with $1 \mathrm{CPU}$;

- Test Case 2: VM instance with 2 CPUs; and

- Test Case 3: VM instance with 4 CPUs.

Each VM hosts the Concat and the Sort web service.

\subsection{Testing Procedure}

The client uses SoapUI [23] to generate various server loads. For each message size of $M$ kilobytes each, we realize experiments where the server generates $N$ web service requests per second in a time frame of 60 seconds. The frequency of requests is not constant and we use variance of 0.5 for generating the load. It means that the frequency of generating requests will vary by $N / 2$ in three equal time periods within the given time frame. Each test starts with a case when the server generates $3 \cdot N / 2$ requests per second in a 20 seconds time frame, then in the next time frame of 20 seconds, the server sends $N / 2$ requests per second, and finally in the last time frame of 20 seconds, the server sends $N$ requests per second.

The range of parameters $M$ and $N$ is selected such that web servers in VM instances work in normal mode without replying error messages and avoiding saturation. The experiments are realized by loading the web services with $N=12 ; 100 ; 500 ; 752 ; 1000 ; 1252 ; 1500 ; 1752$ 


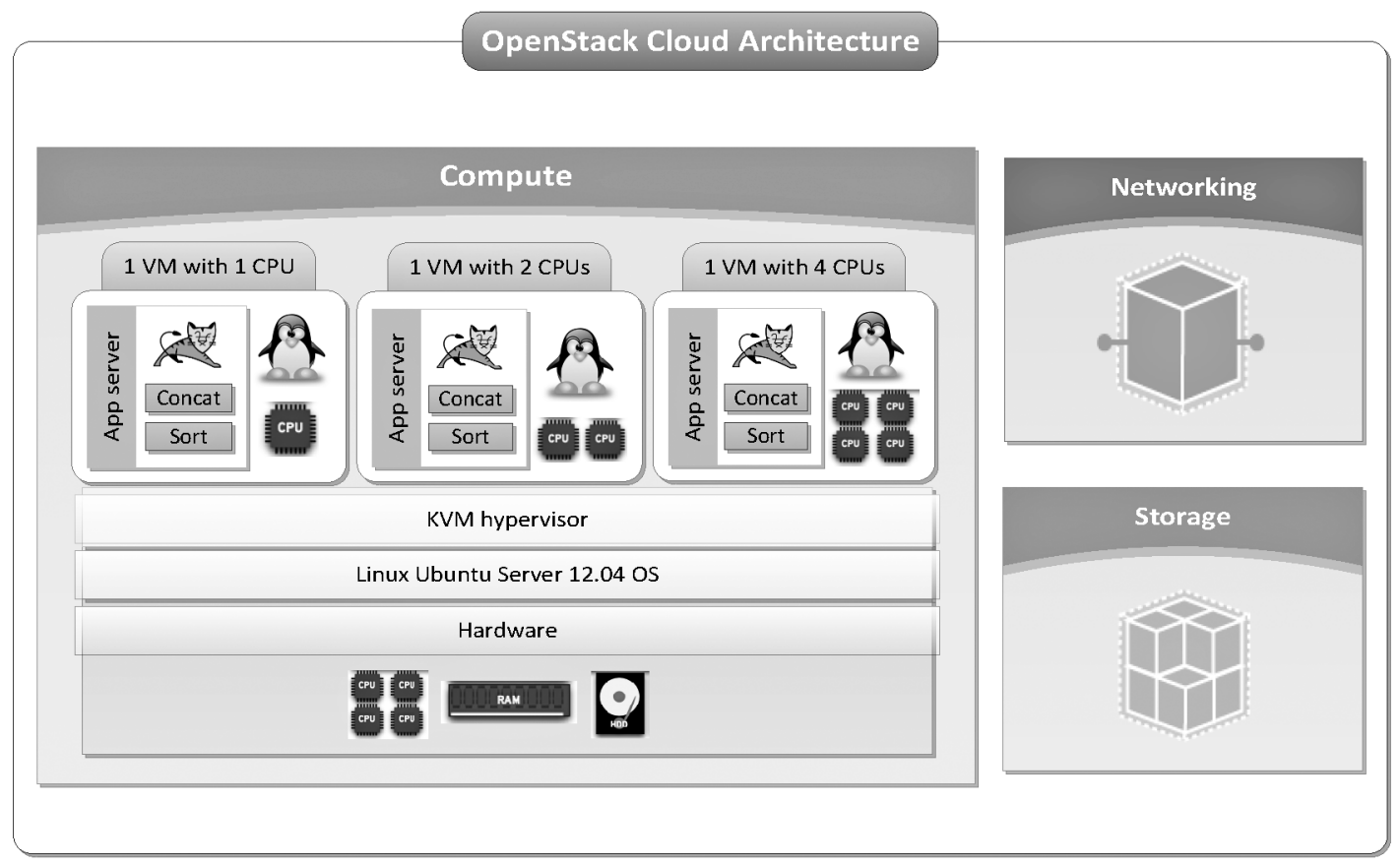

Figure 1. Test cases in the OpenStack cloud architecture.

and 2000 requests per second. We conducted experiments for Concat web service with the following message parameter size $M=0,1, \ldots, 9$ $\mathrm{KB}$, while the experiments for Sort web service are loaded with message parameter size $M=0,1, \ldots, 6 \mathrm{~KB}$.

\subsection{Performance and Cost Measurements}

We measured the server's response time for each different parameter size, $M$, and number of concurrent messages, $N$.

The experiments are realized for each test case, as defined by the environments in 3.3. These measurements will express the cloud's performance. Variable load reflects different time responses, and the response time is measured for each experiment. Since the communication time is less than $1 \mathrm{~ms}$ (around $0.3-0.5 \mathrm{~ms}$ ), the total response time calculated by the SOAPUI is approximately the same as the response time by the server.

We tested three different test cases for $n=1,2$ and 4 cores. For each test case $(n \in\{1,2,4\})$ we calculated the average response time $T(n)$, since the server load was changing with variance 0.5 in the given timeframe of 60 seconds.
Pursuant to our goal of expressing the performance through cost evaluation, in equation (1) we calculate the $\operatorname{cost} C$, that also depends on the total number of used cores $n$.

$$
C(n)=T(n) \cdot n
$$

The goal of this research is to find out whether the performance is proportional to the number of rented resources, thus to check if the web services' total cost $C(n)$ is the real cost of rented $\mathrm{CPU}$ resources. For this purpose we introduce Relative Cost of the scaling, calculated by (2), (3) and (4), as ratio of costs for test cases, comparing VMs with 2 and 1 CPU cores; VMs with 4 and 1 CPU cores; and VMs with 4 and 2 CPU cores, correspondingly.

$$
\begin{aligned}
& R_{21}=\frac{C(2)}{C(1)} \\
& R_{41}=\frac{C(4)}{C(1)} \\
& R_{42}=\frac{C(4)}{C(2)}
\end{aligned}
$$

An ideal expectation will be the proportional scaling, i.e., when $R_{21}=2, R_{41}=4$ and $R_{42}=2$. Any deviation from these expectations will lead to new conclusions in this research. 


\section{The Results of the Experiments}

In this section we present the results of the experiments for each web service separately.

\subsection{Performance Analysis for Concat WS}

The memory demanding Concat web service has been hosted in VM instances with 1,2 and 4 CPU cores.

\subsubsection{Test Case 1 - VM with 1 CPU Core}

Figure 2 presents the results for the cloud environment with $1 \mathrm{VM}$ instance with $1 \mathrm{CPU}$ core. According to the results, the response time depends equally on the message parameter size, $M$, and number of concurrent messages, $N$. Figure 2 denotes that $C(1)$ is equal to $T(1)$ because the number of used CPUs is 1 , and if applied in equation in (1), the cost and the response time remain the same.

For a simplified presentation we denote the points in the format $(M, N)$, where $M$ and $N$ refer to both the parameters we previously defined. Thus, the minimum value of $0.003 \mathrm{~s}$ is in the point $(0,12)$, and the maximum value of $0.241 s$ is in the point $(9,1000)$. Considering that the response time proportionally increases as both of the parameters $M$ and $N$ increase, we observe that the minimum value is in the expected point, but the maximum value seems to be an unexpected peak. The average value is $T(1)=0.058 s$.

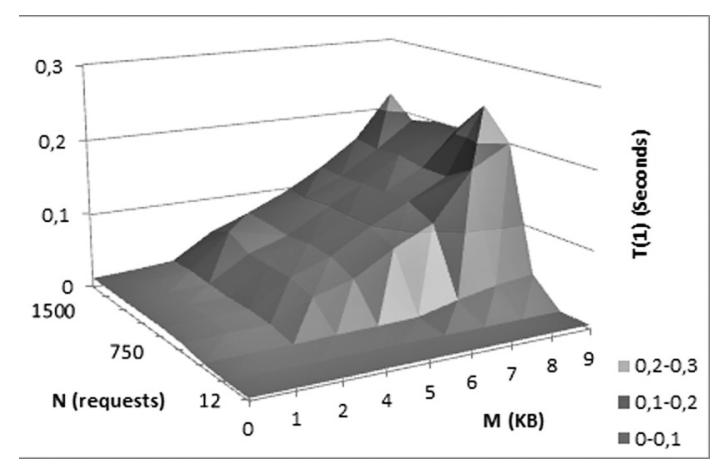

Figure 2. Response time T(1) for Concat WS.

\subsubsection{Test Case 2 - VM with 2 CPU Cores}

The results for the cloud environment with 1 VM instance with 2 CPU cores are presented in Figure 3. The minimum value of $0.002 s$ is at the point $(0,100)$, whereas the maximum value of $0.059 \mathrm{~s}$ is again at the point $(9,1000)$. According to the average value of $T(2)=0.016 \mathrm{~s}$, we assume that the response time has decreased 3.6 times in comparison to the cloud with $1 \mathrm{VM}$ instance with $1 \mathrm{CPU}$. In the next section, where we perform cost analysis, we assume that the performance gain of a VM with 2 cores over a $\mathrm{VM}$ with one core has value of 3.6.

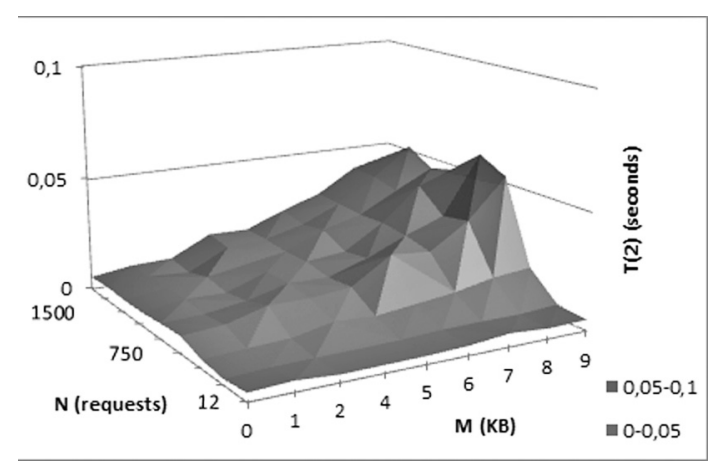

Figure 3. Response time T(2) for Concat WS.

\subsubsection{Test Case 3 - VM with 4 CPU Cores}

Figure 4 depicts the results for the cloud environment with $1 \mathrm{VM}$ instance with $4 \mathrm{CPU}$ cores. The minimum value of $0.003 s$ at $(0,12)$ shows that for small number of concurrent messages and small message parameter size, there is minor variation in the response time in spite of the increased number of resources. The maximum value of $0.031 \mathrm{~s}$ is at the point $(9,1750)$, and the average value is $T(4)=0.012 s$ which means that the response time decreased 4.8 times compared to the cloud VM with 1 core, i.e. the performance gain of a VM with $4 \mathrm{CPU}$ cores compared to a VM with $1 \mathrm{CPU}$ core is 4.8 .

We also calculated the performance gain of a VM with 4 CPU cores over a VM with $2 \mathrm{CPU}$ cores to be $\mathbf{1 . 3}$. 


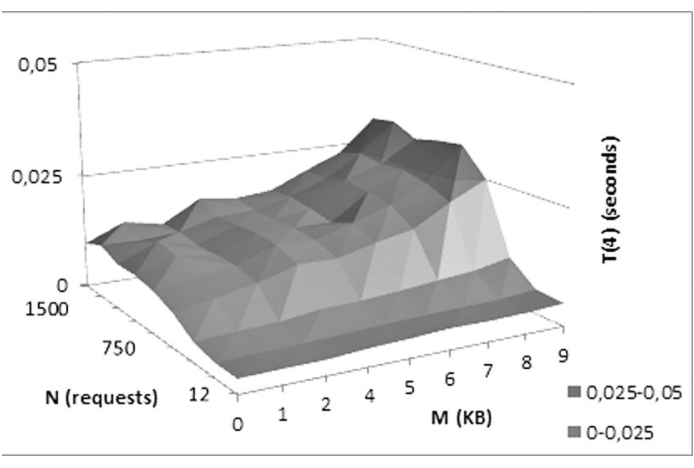

Figure 4. Response time T(4) for Concat WS.

\subsection{Performance Analysis for Sort WS}

The memory and CPU demanding Sort web service has been hosted in VM instances with 1,2 and 4 CPU cores.

\subsubsection{Test Case 1 - 1 Core}

Figure 5 depicts the results for the response time $T(1)$, i.e. VM instance with $1 \mathrm{CPU}$ core.

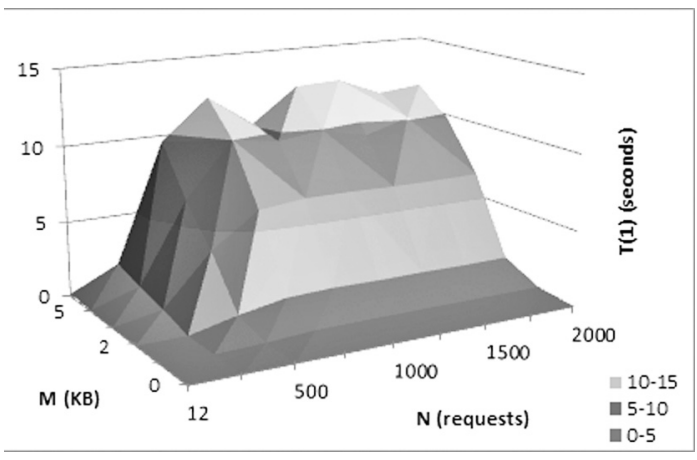

Figure 5. Response time T(1) for Sort WS.

Since the number of cores is $1, T(1)$ is equal to $C(1)$. The response time (cost) proportionally increases with the load, and it depends more on the message size $M$ than on the number of concurrent messages $N$. This can be explained by the fact that Sort is computation intensive and spends more CPU time when processing large messages. The minimum value of $0.003 \mathrm{~s}$ is in the point $(0,100)$, and the maximum value of $12.56 \mathrm{~s}$ is in the point $(6,1500)$. Considering the fact that there is a little variability in the response time for the values of the parameter $N$, we find the minimum and maximum points to be within the expected limits. Unexpected peaks are detected in $(5,750)$ and $(6,750)$. The average value is $T(1)=3.75 \mathrm{~s}$.

\subsubsection{Test Case 2 - 2 Cores}

The results for $T(2)$ are presented in Figure 6. The maximum value detected in this case is $8.7 \mathrm{~s}$, again at the point $(6,1500)$, and the minimum value is $0.002 s$ at $(0,12)$. Considering the average value of $T(2)=2.2 s$, we assume that the $T(2)$ has decreased 1.7 times compared to $T(1)$. Otherwise, it also proportionally increases with the load, depending more on $M$ than $N$.

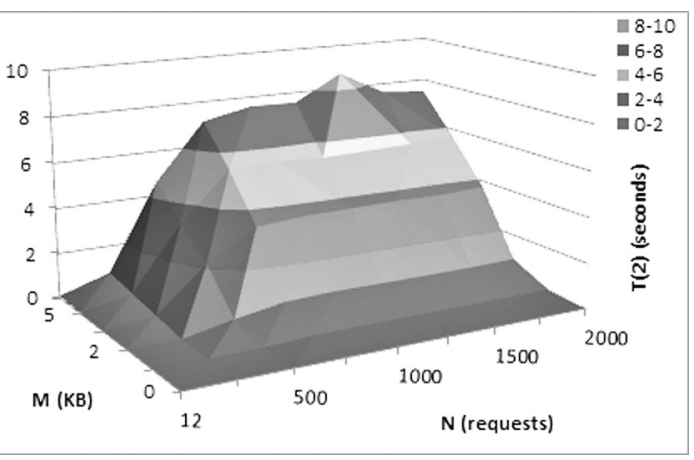

Figure 6. Response time T(2) for Sort WS.

\subsubsection{Test Case 3 - 4 Cores}

Figure 7 presents the results for the response time $T(4)$. The minimum value of $3.02 s$ at $(0,12)$ disproves the scalability property of the response time as the number of cores used increases. However, this phenomenon occurs only for $M=0 K B$. The maximum value of $6.04 \mathrm{~s}$ is at the point $(6,1750)$, and the average value is $T(4)=1.64 \mathrm{~s}$ which means that $T(4)$ decreased 2.3 times compared to $T(1)$, and $\mathbf{1 . 3 4}$ times compared to $T(2)$.

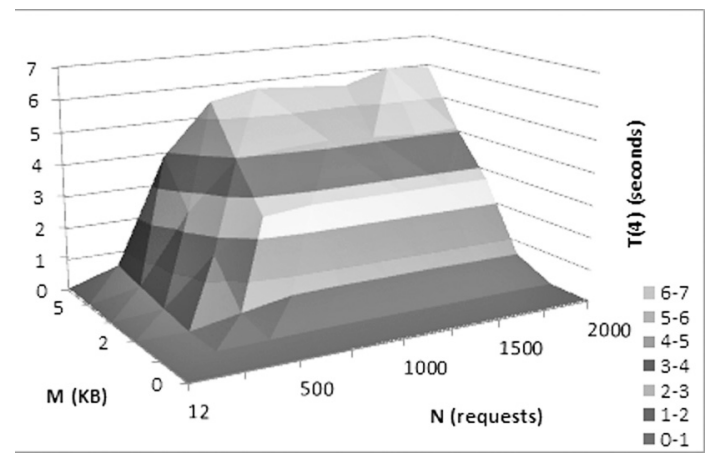

Figure 7. Response time T(4) for Sort WS. 


\section{Evaluation of Cost and Performance}

In this section we perform evaluation of cost and performance for realized experiments and obtained results.

\subsection{Cost Analysis for Concat Web Service}

In order to derive conclusions about the sufficient trade-off between the cost and the gained performance, we used (1) to calculate the customer's cost for the resources.

By observing the average response time decrease in Section 4.1, we have concluded that scaling up the resources $n$ times, where $n$ is the number of cores, provides performance gain, even more than $n$ times. Hereupon, we aim to find an answer for our research problem, i.e. whether the cost for the resources is equal to the performance gain, moreover, whether there is a region where customers pay less than they gain.

The default cost per core is the cloud environment with one core, i.e., the results from the cloud environment with $1 \mathrm{VM}$ instance with 1 CPU as presented in Figure 2.

The relative ratio between the cost for the cloud VM with 2 CPUs and cloud VM with $1 \mathrm{CPU}$, calculated by (2) is depicted in Figure 8. As cost threshold we used the average response time decrease of 3.6, since in Section 4.1 we showed that the consumer achieves 3.6 times more performance on average when migrating from VM with 1 CPU to VM with 2 CPUs. Thus, if the cost transcends this value, a customer is considered to pay more than he gets. Interestingly, we observe that the customer's costs for resources remain far beyond the threshold value.

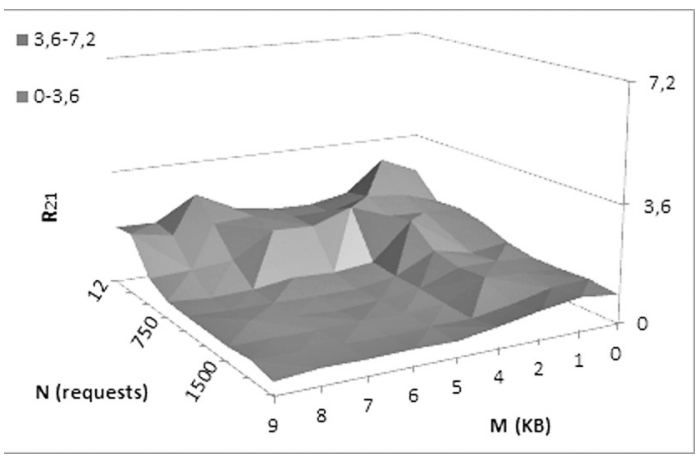

Figure 8. Relative cost $R_{21}$ for Concat WS.
Figure 9 depicts the proportion of the cost for the cloud VM with 4 CPUs and the cost for a cloud VM with 1 core, calculated by (3). The results also show that, for any message parameter size and number of concurrent messages, the customer's costs are much lower than the performance advantage of $\mathbf{4 . 8}$ times. Interestingly, incrementing the number of concurrent messages $N$ and the message size $M$ decreases the cost in both scenarios.

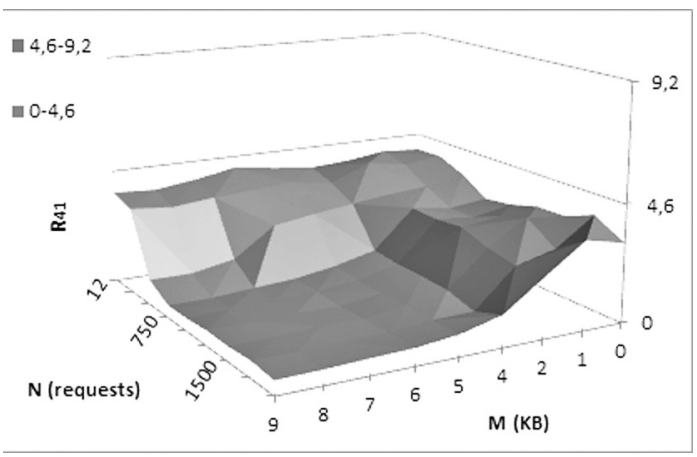

Figure 9. Relative cost $R_{41}$ for Concat WS.

Eventually, we compared the cost between the cloud VM instance with 4 CPUs and cloud VM instance with 2 CPUs, calculated by (4). The aim of this analysis is to discover whether the customer will make a good decision demanding 2 more CPUs once he has used 2 cores. When presenting the results on Figure 10, we used 1.3 value as a threshold which we obtained from the results in Section 4.1 where we concluded that the performance gain is 1.3 times for $\mathrm{VM}$ with 4 cores in comparison to VM with 2 cores. However, the results show that there are small regions where the customer pays less than he gets, thus, it is not worthwhile renting 2 more cores once the customer has used 2 cores.

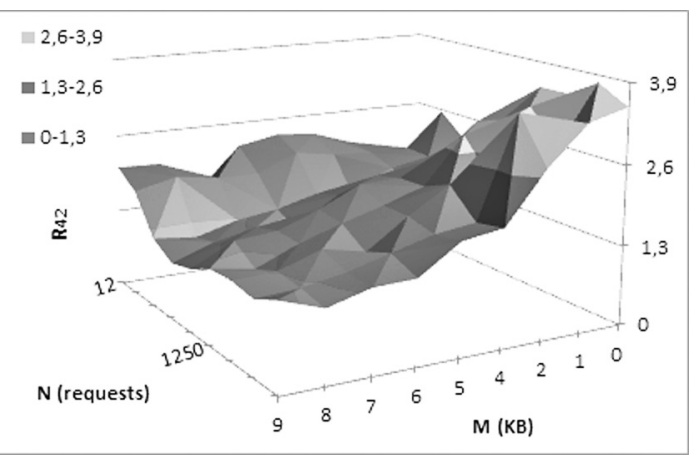

Figure 10. Relative cost $R_{42}$ for Concat WS. 


\subsection{Cost Analysis for Sort Web Service}

Using the values for response time in (1), we calculate the customer's cost for rented resources and define the best trade-off between the cost and the gained performance.

Considering the average response time decrease in Section 4.2, we concluded that scaling up the resources $n$ times does not provide equal performance scale. Hereupon, we aim to investigate whether the cost for resources is nearly equal to the performance gain, more precisely, if the customers pay as much as the gained performance.

To compare the scaling with factor 2 , we analyze the results for relative cost $R_{21}$ depicted in Figure 11. As a cost threshold we used the average performance decrease of $\mathbf{1 . 7}$, thus, if the cost transcends this value, a customer is considered to pay more than he gets. We observe that the cost is within the limits except for the load with small values of $M$ and $N$. We explain this with the fact that web server needs more time to schedule the small number of small tasks instead of executing them.

The relative cost $R_{41}$ is depicted in Figure 12 . The results show that the customer pays more than he gets when one or both parameters $M$ and $N$ are small. For greater load the customer will obtain the performance of the resources he pays.

The results for the relative cost $R_{42}$ are depicted in Figure 13. The threshold value for scaling the resources from scaling factor 2 to 4 is $\mathbf{1 . 3 4}$, but the relative cost $R_{42}$ is much greater than the threshold for almost all values of parameters $M$ and $N$. Although there is a small region where the relative cost is smaller than the threshold, its value is still near the threshold.

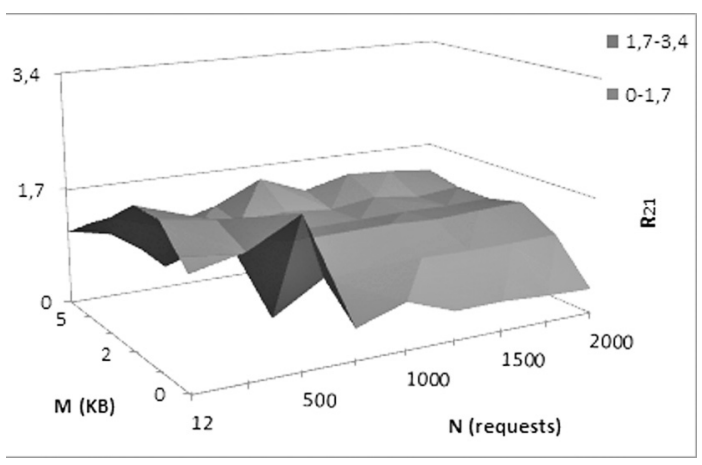

Figure 11. Relative cost $R_{21}$ for Sort WS.

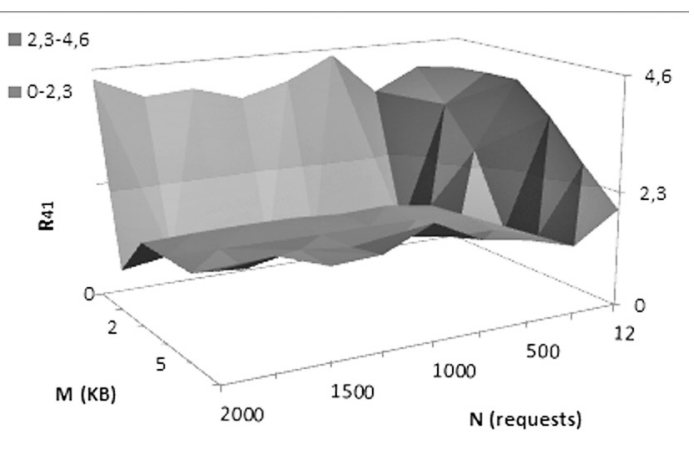

Figure 12. Relative cost $R_{41}$ for Sort WS.

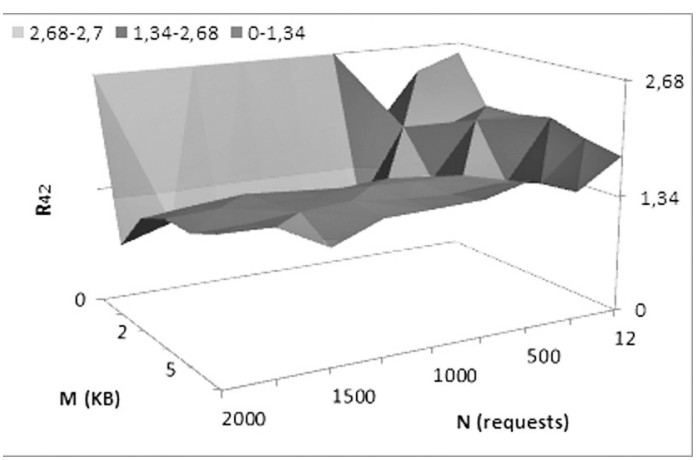

Figure 13. Relative cost $R_{42}$ for Sort WS.

\section{Discussion}

In this section we compare the results from the performance and cost analysis of the two web services.

\subsection{Response Time Comparison}

When discussing performance disproportion to the scaled resources, the Concat web service performs better for both cloud environments with VM instance with 2 and 4 CPUs respectively, defined in Section 3.3. Therefore, when the Concat web service is hosted in cloud VM instance with 2 cores, the response time decreases 3.6 times in comparison to the situation when it is hosted in cloud VM instance with 1 core. When the same web service is hosted in cloud environment with VM instance with 4 cores, according to the average response time values, the performance improves 4.8 times than when it is hosted in cloud VM with 1 core. On 
the other hand, for the Sort web service, the response time decreases 1.7 times when the CPU scales to 2 cores, and 2.3 times if the number of CPUs is 4. Obviously, the performance improvement depends on the type of the web service used. For a memory demanding web service, customers will gain higher performance when renting more resources, whereas for computation intensive web service, the increasing performance factor is much smaller, but it also has the affinity to rise.

\subsection{Cost Analysis Comparison}

In this section we proceed to discuss the tradeoff between the cost and the performance. We observe that the Concat web service cost, while hosted on cloud VM instance with 2 CPUs, never transcends the 3.6 threshold. The cost analysis of the Concat web service hosted in cloud VM instance with 4 cores, using the average response time decrease value of 4.8 , also shows that pay-per-usage model is convenient for the customers. Even though, the computation intensive Sort web service shows less performance gain when scaling the resources, there are only few regions where the cost transcends the performance.

The overall comparison results show that the compromise between the cost and the performance is positive, which means the customers will gain as much performance as they pay with negligible exceptions.

\section{Conclusion and Future Work}

CSPs' pay-per-usage model offers linearly scalable charging to the acquired resources. We assume that the performance also scales to the pay-per-usage model and in this paper we performed a series of experiments to investigate in which case the customer would make the best trade-off between the performance and the expenses.

Observing all scenarios, we conclude that the Concat web service, while hosted on 2 CPUs, provides 3.6 time better performance than when hosted on $1 \mathrm{CPU}$, which is the best case from all test cases. Moreover, it also provides the lowest cost in comparison to the gained performance. On the other hand, the worst results are obtained when the customer migrates from 2 CPUs to 4 CPUs, i.e., the cost is much above the performance benefit.

In comparison to Concat, Sort web service shows less performance gain when scaling the resources, however, there are only few regions where the cost transcends the performance.

By performing the analyses of both web services, we confirmed that the cost-performance relation still depends on the web services' characteristics. Overall results show that for a memory demanding web service the customer will gain maximum performance for the particular investment, but adding more characteristics to the web service this performance gain decreases. However, generally, the performance gain stays positive.

This intrigues us to extend our research in the future and to include more web services that depend on different characteristics. Furthermore, we will perform the same analysis in a multitenant cloud, since although virtualization has facilitated resource efficiency in servers, currently, performance interference effects still occur in virtual environments where the same $\mathrm{CPU}$ cache and translation lookaside buffer (TLB) hierarchy are shared by multiple VMs [24].

\section{References}

[1] R. Buyya, C. S. Yeo, S. Venugopal, Marketoriented cloud computing: Vision, hype, and reality for delivering it services as computing utilities. In High Performance Computing and Communications, HPCC'08. 10th IEEE International Conference on, (2008), pp. 5-13.

[2] B. SosINSKY, Cloud computing bible. John Wiley \& Sons, 2010.

[3] R. Grossman, The case for cloud computing. IT professional,11(2) (2009), 23-27.

[4] D. DURKEE, Why cloud computing will never be free. Quеие, 8(4) (2010), p. 20.

[5] Microsoft, Windows Azure, July 2013. [Online]. Available:

http://www.windowsazure.com/pricing/

[6] Google, Compute Engine, July 2013. [Online]. Available:

http://cloud.google.com/pricing/ 
[7] Amazon, EC2, July 2013. [Online]. Available: http://aws . amazon.com/ec2/

[8] M. Simjanoska, G. Velkoski, S. Ristov, M. GuSEV, Does the performance scale the same as the cost in the cloud? In Information Technology Interfaces, Proceedings of the ITI 2013 35th International Conference on, IEEE Conference Proceedings, (2013), pp. 83-88.

[9] A. Berl, E. Gelenbe, M. Di Girolamo, G. GiULiani, H. De MeER, M. DANG, K. Pentikousis, Energy-efficient cloud computing. The Computer Journal, 53(7) (2010), 1045-1051.

[10] J. Baliga, R. Ayre, K. Hinton, R. Tucker, Green cloud computing: Balancing energy in processing, storage, and transport. Proceedings of the IEEE, 99(1) (2011), 149-167.

[11] A. Beloglazov, R. BuYyA, Energy efficient resource management in virtualized cloud data centers. In Proceedings of the 2010 10th IEEE/ACM International Conference on Cluster, Cloud and Grid Computing, ser. CCGRID '10, (2010), pp. 826-831.

[12] Y. LEE, A. ZOMAYA, Energy efficient utilization of resources in cloud computing systems. The Journal of Supercomputing, $\mathbf{6 0}$ (2) (2012), 268-280.

[13] Q. Zhang, L. Cheng, R. Boutaba, Cloud computing: state-of-the-art and research challenges. Journal of Internet Services and Applications, 1(1) (2010), 7-18.

[14] M. De Assunção, A. Di Costanzo, R. Buyya, Evaluating the cost-benefit of using cloud computing to extend the capacity of clusters. In Proceedings of the 18th ACM international symposium on High performance distributed computing ACM, (2009), pp. 141-150.

[15] A. AndrZejaK, D. Kondo, S. Yi, Decision model for cloud computing under sla constraints. In Modeling, Analysis \& Simulation of Computer and Telecommunication Systems (MASCOTS), 2010 IEEE International Symposium on, (2010), pp. 257-266.

[16] H. Truong, S. Dustdar, Composable cost estimation and monitoring for computational applications in cloud computing environments. Procedia Computer Science, 1(1) (2010), 2175-2184.

[17] D. Kondo, B. JaVAdi, P. Malecot, F. CAPPELlo, D. ANDERSON, Cost-benefit analysis of cloud computing versus desktop grids. In Parallel \& Distributed Processing, 2009. IPDPS 2009. IEEE International Symposium on, (2009), pp. 1-12.

[18] M. GusEv, S. Ristov, The optimal resource allocation among virtual machines in cloud computing. In CLOUD COMPUTING 2012, The Third International Conference on Cloud Computing, GRIDs, and Virtualization, (2012), pp. 36-42.

[19] S. Ristov, G. Velkoski, M. GuseV, K. KJIROSKI, Compute and memory intensive web service performance in the cloud. In ICT Innovations 2012. Springer Berlin/Berlin Heidelberg, 2013, vol. AISC 257, pp. 215-224.
[20] M. Gusev, S. Ristov, G. Velkoski, M. SimJANOSKA, Optimal resource allocation to host web services in cloud. In Cloud Computing (CLOUD), 2013 IEEE 6th International Conference on, ser. CLOUD 2013, June 2013, pp. 948-949.

[21] OPENSTACK, Openstack cloud software, July 2013. [Online]. Available: http://openstack.org

[22] M. JURIC, I. RozMAN, B. BRUMEN, M. COLNARIC, M. HERICKO, Comparison of performance of web services, WS-Security, RMI, and RMI-SSL. Journal of Systems and Software, 79(5) (2006), 689700.

[23] SoApUI, Functional testing tool for web service testing, July 2013. [Online]. Available: http://www . soapui.org/

[24] F. HU, M. QIU, J. Li, T. GRANT, D. TAYLOR, S. MCCALEB, L. Butler, R. HAMnER, A review on cloud computing: Design challenges in architecture and security. Journal of Computing and Information Technology, 19(1) (2011), 25-55.

Received: July, 2013 Revised: September, 2013 Accepted: September, 2013

Contact addresses:

Monika Simjanoska Faculty of Computer Sciences and Engineering Ss. Cyril and Methodius University Skopje Macedonia e-mail: m.simjanoska@gmail.com

Marjan Gusev Faculty of Computer Sciences and Engineering Ss. Cyril and Methodius University Skopje Macedonia e-mail: marjan.gushev@finki.ukim.mk

Sasko Ristov Faculty of Computer Sciences and Engineering Ss. Cyril and Methodius University Skopje Macedonia e-mail: sashko.ristov@finki.ukim.mk

Goran Velkoski Faculty of Computer Sciences and Engineering Ss. Cyril and Methodius University Skopje

Macedonia e-mail: velkoski.goran@gmail.com

MoniKA SIMJANOSKA was born in Zagreb, Croatia, in 1988. She received her BSc degree in Computer Science and Engineering in 2012 from the Ss. "Cyril and Methodius" University - Skopje, Macedonia. Since 2010, she has been a Teaching Assistant at the Ss. "Cyril and Methodius" University - Skopje, Macedonia. Recently, she works on her master thesis in the field of bioinformatics in the cloud. The research field of Monika Simjanoska is cloud computing's performance, machine learning in the cloud and biological intelligent systems engineering. She is author and co-author of more than 15 research papers. 
MARJAN GuSEV is a professor at the Ss. "Cyril and Methodius" University, Faculty of Computer Science and Engineering, Skopje, Macedonia. He has published more than 70 papers in parallel computing related journals and is author of more than 300 research articles, presentations, invited talks and technical reports. His areas of interest include computer architecture, parallel processing, computer networks, internet, and cloud computing. He was awarded with Best Scientist Award at the University Ss. "Cyril and Methodius", and with the IEEE EDUCON 2013 Best Paper Award.

SASKO RISTOV was born in Kavadarci, Macedonia, in 1976. He received his Ph.D. degree in 2012. Since 2013 he is Assistant Professor at the Ss. "Cyril and Methodius" University - Skopje, Macedonia. He has authored more than 70 research articles during his relatively short scientific research. His research interests include performance and security of cloud computing, implementing the cloud features in education, especially hardware, network and parallel and distributed processing courses. Sasko Ristov was a recipient of the University Ss. "Cyril and Methodius" - Skopje, Macedonia the Best Scientist Award in 2012, and the IEEE EDUCON 2013 Best Paper Award.

GORAN VELKOSKI was born in Skopje, Macedonia, in 1990. He received his BSc degree in Computer Science and Engineering in 2012. Since 2012, he has been a Teaching Assistant at the Ss. "Cyril and Methodius" University - Skopje, Macedonia. Recently, he works on his master thesis in the field of cloud computing. Goran Velkoski is author and co-author of more than 20 research papers and has received the IEEE EDUCON 2013 Best Paper Award in the field of "Innovative Materials, Teaching and Learning Experiences in Engineering Education". 